\title{
Risk prediction for coronary heart disease by a genetic risk score - results from the Heinz Nixdorf Recall study
}

Sonali Pechlivanis ${ }^{1 *}$ (D, Nils Lehmann ${ }^{1}$, Per Hoffmann ${ }^{2,3}$, Markus M. Nöthen ${ }^{2}$, Karl-Heinz Jöckel ${ }^{1}$, Raimund Erbel ${ }^{1}$ and Susanne Moebus ${ }^{1,4}$

\begin{abstract}
Background: A Genetic risk score for coronary artery disease (CAD) improves the ability of predicting coronary heart disease (CHD). It is unclear whether i) the use of a CAD genetic risk score is superior to the measurement of coronary artery calcification (CAC) for CHD risk assessment and ii) the CHD risk assessment using a CAD genetic risk score differs between men and women.

Methods: We included 4041 participants (age-range: 45-76 years, 1919 men) of the Heinz Nixdorf Recall study without CHD or stroke at baseline. A standardized weighted CAD genetic risk score was constructed using 70 known genetic variants. The risk score was divided into quintiles (Q1-Q5). We specified low (Q1), intermediate (Q2Q4) and high (Q5) genetic risk groups. Incident CHD was defined as fatal and non-fatal myocardial infarction, stroke and coronary death. The association between the genetic risk score and genetic risk groups with incident CHD was assessed using Cox models to estimate hazard ratios (HR) and 95\%-confidence intervals (Cl). The models were adjusted by age and sex (Model1), as well as by established CHD risk factors (RF) and CAC (Model2). The analyses were further stratified by sex and controlled for multiple testing.

Results: During a median follow-up time of $11.6 \pm 3.7$ years, 343 participants experienced CHD events (219 men). Per-standard deviation (SD) increase in the genetic risk score was associated with 18\% increased risk for incident CHD (Model1: $p=0.002$ ) which did not change after full adjustment (Model2: HR= 1.18 per-SD $(p=0.003)$ ). In Model2 we observed a $60 \%$ increased CHD risk in the high $(p=0.009)$ compared to the low genetic risk group. Stratifying by sex, only men showed statistically significantly higher risk for $\mathrm{CHD}$ (Model2: HR=1.23 per-SD ( $p=0.004$ ); intermediate: $H R=1.52(p=0.04)$ and high: $H R=1.88(p=0.008))$ with no statistically significant risk observed in women.

Conclusion: Our results suggest that the CAD genetic risk score could be useful for CHD risk prediction, at least in men belonging to the higher genetic risk group, but it does not outbalance the value of CT-based quantification of CAC which works independently on both men and women and allows better risk stratification in both the genders.
\end{abstract}

Keywords: Coronary heart disease, Coronary artery disease, Coronary artery calcification, Genetic risk score, Cohort study

\footnotetext{
* Correspondence: sonali.pechlivanis@uk-essen.de

${ }^{1}$ Institute for Medical Informatics, Biometry and Epidemiology, University

Hospital of Essen, University Duisburg-Essen, Essen, Germany

Full list of author information is available at the end of the article
}

(c) The Author(s). 2020 Open Access This article is licensed under a Creative Commons Attribution 4.0 International License, which permits use, sharing, adaptation, distribution and reproduction in any medium or format, as long as you give appropriate credit to the original author(s) and the source, provide a link to the Creative Commons licence, and indicate if changes were made. The images or other third party material in this article are included in the article's Creative Commons licence, unless indicated otherwise in a credit line to the material. If material is not included in the article's Creative Commons licence and your intended use is not permitted by statutory regulation or exceeds the permitted use, you will need to obtain permission directly from the copyright holder. To view a copy of this licence, visit http://creativecommons.org/licenses/by/4.0/. The Creative Commons Public Domain Dedication waiver (http://creativecommons.org/publicdomain/zero/1.0/) applies to the data made available in this article, unless otherwise stated in a credit line to the data. 


\section{Background}

Coronary heart disease (CHD) is one of the leading causes of deaths with over 1.8 million and 836,546 deaths in European Union as well as in the USA respectively, at the estimated annual cost of $€ 210$ billion in the European Union and $\$ 329.7$ billion in the USA [1, 2]. Within the European Union, CHD is the second cause of death with around $24 \%$ death among men and $17 \%$ among women under 65 years [1]. The European Association for Cardiovascular Prevention and Rehabilitation and the American College of Cardiology/American Heart Association (ACC/AHA) recommended practice guidelines to reduce the risk of $\mathrm{CHD}$ events [3, 4]. The association of several risk factors (RF) with CHD has led to development of a couple of CHD risk prediction models. These models incorporate information about age, sex, systolic blood pressure, cholesterol, smoking habits and diabetes mellitus to predict CHD risk. The quantification of coronary artery calcification (CAC) has been shown to allow better risk prediction of future CHD events then traditional risk prediction models [57]. Currently, the Multi Ethnic Study of Atherosclerosis (MESA) investigating group has developed a useful tool that includes CAC as well as established RFs to predict CHD risk [6].

The heritability of CHD has been estimated to be between 40 and $60 \%$ based on family, twin and genomewide association studies (GWAS) [8-10]. Genetic risk scores based on single nucleotide polymorphisms (SNPs) that are associated with coronary artery disease (CAD) have been shown to improve the risk prediction of CHD [11-16]. Most of these genetic studies used Framingham or established RFs to estimate the CHD risk. A recent study from MESA could show an association between CAD genetic risk score and incident CHD only in men [11]. However, it has not been fully assessed i) whether the use of a CAD genetic risk score is superior to the measurement of CAC for CHD risk assessment and ii) whether the CHD risk assessment using a CAD genetic risk score differs between men and women.

\section{Methods}

\section{Study population}

Heinz Nixdorf Recall study is a population based cohort study consisting of 4814 participants, aged 45 to 75 years (50\% women) at baseline. The study participants were randomly selected from the registration lists of the densely populated Ruhr metropolitan cities in Germany (residents of Essen, Bochum, and Mülheim an der Ruhr) between December 2000 and August 2003. The rationale and design of the study were described in detail previously [17]. The participants were re-invited for first and second follow-up examination taken place approximately 5 and 10 years after the baseline examinations.
Participants with prior CHD (coronary artery bypass surgery and/or interventional revascularization, history of prior myocardial infraction and stroke) $(n=327)$ at baseline were excluded from the present study.

\section{Coronary heart disease}

Incidental $\mathrm{CHD}$ was the primary end-points of our study. A pre-defined study criteria were used to clearly document the incidental CHD [18]. Hospital and nursing home records along with ECGs, laboratory values and pathology reports were collected for all the primary end-points [19]. Death certificates and interviews with general practitioners, relatives and eye witness were also obtained. Medical reports were further collected for all the reported end points [20]. All the documents were studied by an external end-point committee who were blinded for RF status. The end-point committee further classified the end points at separate regular meetings twice a year. CHD was defined as fatal and non-fatal myocardial infarction, stroke and coronary death. All the incident CHD that occurred between the baseline and third examination $(n=343,8.5 \%)$ was included in the present study.

\section{Assessment of coronary artery calcification}

Non-enhanced electron-beam scan (C-100 or C-150 scanner, GE Imatron, San Francisco, CA, USA), was used to assess CAC at baseline [17]. The prospective ECG-triggering was performed at $80 \%$ of the RRinterval. At an image acquisition time of $100 \mathrm{~ms}$, contiguous $3 \mathrm{~mm}$ thick slices from the pulmonary bifurcation to the apex of the heart were then obtained in both the scans [21]. CAC was quantified using the methods of Agatston et al. [22]. The total CAC score was computed which comprised of all the calcified lesions in the coronary artery system. Virtuoso workstation (Siemens Medical Solutions, Forchheim, Germany) was used to perform the analyses. The results of CT scan were not disclosed to the study center or to the participants. We further categorized CAC into two groups i.e. $\mathrm{CAC}=0$ (absence of $\mathrm{CAC}$ ) and $\mathrm{CAC}>0$ (presence of CAC).

\section{Cardiovascular risk factors}

Cardiovascular RFs were recorded at baseline. As described previously smoking status (smokers (defined as current or past smokers) and non-smoker) was assessed in detail [23]. Current regular use of medication which included antihypertensive or lipid lowering medications was recorded in a standardized assessment of medications. We calculated the body mass index (BMI) as weight divided by height square $\left(\mathrm{kg} / \mathrm{m}^{2}\right)$. As described previously, resting blood pressure was measured using an automated oscillometric blood pressure device 
(Omron, HEM-705CP-E) with the participants seated. Of the three measurements the mean of the second and third value was calculated [24]. Serum triglycerides, low density lipoprotein (LDL)-cholesterol and high density lipoprotein (HDL)-cholesterol values were determined using the standardized enzymatic methods (ADVIA 1650, Siemens Medical Solutions, Erlangen, Germany). Diabetes was defined as either of 4 criteria: (1) participants reported a history of clinically diagnosed diabetes, (2) participants took glucose-lowering medications, (3) participants had fasting glucose levels (FPG) of greater than $125 \mathrm{mg} / \mathrm{dL}$, or (4) participants had non-fasting glucose levels of $200 \mathrm{mg} / \mathrm{dL}$ or greater [25].

\section{Genotyping and genetic risk score}

In the Heinz Nixdorf Recall study, 4371 participants were genotyped using Illumina GWAS chips (Omni1, OmniExpress, OmniExpress1, HumanCoreExome (v1.0 and v1.1); Illumina, San Diego, USA) and 4518 participants using Metabochip [26, 27]. Quality control (QC) was performed separately for all the chips at the subject level and then on SNPs before imputing each chip with IMPUTE v2.3.1 with reference data from 1000 Genomes Phase 1 release March 2012 for the Metabochip and 1000 Genomes Phase 3, release October 2014 for all the other chips $[26,28,29]$. At subject level, the QC involved sex-, ethnicity- and relatedness-checks. We excluded the participants if HET $>5$ standard deviations of the mean, $>5 \%$ missing genotype data and outliers identified by principle component analysis. Thereafter, we excluded the SNPs with a minor allele frequency (MAF) $<1 \%$, a missing genotype frequency $>5 \%$ or a deviation from Hardy-Weinberg Equilibrium (HWE) $\left(p<10^{-5}\right.$ ). GTOOL v0.7.5 (threshold $\geq 0.8$ ) was next used to convert the imputed data into the PLINK ped format.

For this study, 70 SNPs were selected based on the published CAD GWAS $\left(p \leq 5 \times 10^{-8}\right)$ to construct the genetic risk score [30-33] and recently published by Pechlivanis et al. [34]. The average weighted genetic risk score for each individual was constructed by using the risk estimate (transformed by natural $\log$ ) from the published CAD GWAS and multiplying by the number of CAD risk alleles; these products were then summed up. The summed up product was then divided by the number of SNPs $(n=70)$. The allelic scoring routine in PLINK was used to calculate the genetic risk score [35]. The expected value based on the sample allele frequency was imputed, if the genotype in the score for a particular individual was missing. The mean (0.03) and standard deviation (SD; 0.003 ) of the study population were used to standardize the genetic risk score to have a mean of zero and unit variance. Genetic risk was then analyzed per-SD of the standardized genetic risk score. For our analyses we used 4041 participants having information on genetic risk score, sex, age and CAC at baseline.

\section{Statistical analysis}

We calculated the Kaplan-Meier estimates of event-free survival probabilities in all the study participants as well as participants stratified by sex which was evaluated using a log-rank test of trend. The association of the genetic risk score with incident $\mathrm{CHD}$ was assessed with multivariable Cox proportional hazards regression to calculate the adjusted hazard ratios and corresponding 95\% confidence intervals. The models were first adjusted for age and sex and the established RF adjusted model consisted of age, sex, systolic blood pressure, antihypertensive medication, smoking, LDL-cholesterol, HDLcholesterol, lipid lowering medication, BMI, diabetes and CAC. The genetic risk score was analyzed as a continuous variable defined by per-SD of the standardized CAD genetic risk score and as ordinal scaled distribution to define genetic risk groups using quintiles (Q1-Q5). Q1 characterizes the low, Q2-Q4 the intermediate and Q5 the high genetic risk group.

The association between the genetic risk score and CAC was assessed in a logistic regression model. We excluded the participants with any missing data from the respective analysis.

Uno's concordance statistics was used to evaluate the risk predictions [36]. Additionally, to find the best model to describe the relationship between genetic risk score and incident CHD we used Akaike's information criterion (AIC) [37]. In our calculations, the lower the AIC value, the better was the model.

We controlled for multiple testing at 5\% for our primary question relating the association of the genetic risk score per-SD with incident CHD in all, men and women study participants for the age and sex as well as RF plus CAC adjusted models. Accordingly, we corrected for 6 statistical tests that translate into $\alpha_{B F}=0.008$ using the Bonferroni procedure.

In order to test the hypothesis of a causal association between CAC and CHD, a Mendelian randomization analysis using CAD GRS as an instrumental variable was carried out [38]. In our analysis, genetically determined CAC (as predicted by the CAD GRS) was regressed against CHD. The inverse-variance weighted (IVW) method was used using the summary statistics (beta and standard error i.e., by scaling the natural logarithm of the OR) for the associations of the CAD GRS with CAC (exposure) and (beta and standard error i.e., by scaling the natural logarithm of the HR) CHD (outcome) from our study. The analyses were first carried out using all the study participants and then stratified by sex.

The continuous data are presented as mean \pm SD or median (first quartile: $\mathrm{q}(25)$, third quartile: $\mathrm{q}(75)$ ) if the 
distribution of data were substantially skewed. We performed the tests for group differences for the continuous data using the Student's $t$ test or the Mann-Whitney $U$ test. Count data are presented as frequency and percentage. The difference in the group was evaluated by the $\chi^{2}$ or the Fisher exact test. The statistical analyses were done using SAS v.9.4.

\section{Results}

\section{Study characteristics}

The baseline characteristics of the Heinz Nixdorf Recall study participants are shown in Table 1 . During a median follow up time of $11.6 \pm 3.7$ years, $343(8.5 \%)$ participants experienced CHD events (219 men). The mean and standard deviation (SD) of the weighted genetic risk score in the Heinz Nixdorf Recall study participants was $0.03 \pm 0.003$. The weighted genetic risk score was statistically significantly $(p=0.006)$ higher in those with events $(0.04 \pm 0.003)$ than without events $(0.03 \pm 0.003)$. CAC $(\log (C A C+1))$ was statistically significantly $(p<0.0001)$ higher in those with events (median (Q1; Q3), 4.8 (2.7; 6.1)) than without events $(2.3(0 ; 4.5))$ (Table 1$)$. Also, the proportion of participants with presence of CAC $(\mathrm{CAC}>0)$ were statistically significantly $(p<0.0001)$ higher in those with events $(86.9 \%)$ than without events (65.9\%). The amount of CAC increased with increase in the genetic risk group. Participants in the high genetic risk group showed the highest $C A C$ values $(\log (C A C+$
1)) (high genetic risk: $3.2(0 ; 5.2)$, intermediate genetic risk: $2.5(0 ; 4.7)$ and low genetic risk: $1.9(0 ; 4.4)$ ) (data not shown). Also, higher CAC was observed in men (high genetic risk: $4.5(2.2 ; 5.8)$, intermediate genetic risk: $4.0(1.7 ; 5.5)$ and low genetic risk: $3.6(0.7 ; 5.0))$ as well as in women (high genetic risk: $1.3(0 ; 4.0)$, intermediate genetic risk: $0.9(0 ; 3.5)$ and low genetic risk: 0.7 $(0,3.3))$ belonging to the higher genetic risk group (data not shown). Kaplan-Meier curves for CHD events are shown in the Additional file Fig. 1 (a) and (b). An effect in men can be detected in the Kaplan-Meier survival curves (Additional file: Fig. 1 (b)), when the nonadjusted data were used.

\section{Genetic risk score and incident coronary heart disease}

After adjusting for age and sex, the CAD genetic risk score was statistically significantly associated with incident CHD (hazard ratios (HR) $=1.18$ per-SD; 95\% confidence interval $[95 \% \mathrm{CI}][1.06 ; 1.31], p=0.002)$ even after adjusting for multiple testing. Further adjusting for established RFs including systolic blood pressure, antihypertensive medication, smoking, LDL-cholesterol, HDLcholesterol, lipid lowering medication, BMI, diabetes and CAC showed similar statistically significant effect $(\mathrm{HR}=$ 1.18 per-SD [1.06; 1.31], $p=0.003$ ) (Fig. 1(a)). In the sex stratified analyses, men showed statistically significant higher risk for incident $\mathrm{CHD}\left(\mathrm{HR}_{\mathrm{men}}=1.25\right.$ per-SD $[1.10 ; 1.42], p=0.001)$ with no statistically significant

Table 1 Characteristics of the Heinz Nixdorf Recall study population

\begin{tabular}{|c|c|c|c|c|}
\hline & $\begin{array}{l}\text { All Events/N } \\
343 / 4041\end{array}$ & $\begin{array}{l}\text { Events N (\%) } \\
343(8.5)\end{array}$ & $\begin{array}{l}\text { No Events N (\%) } \\
3693(91.5)\end{array}$ & $p^{c}$ \\
\hline$\overline{\text { Age (years) }}{ }^{a}$ & $58.9 \pm 7.6$ & $63.5 \pm 7.8$ & $58.9 \pm 7.6$ & $<0.0001$ \\
\hline Men & $1919(47.5)$ & $219(63.9)$ & $1700(46.0)$ & $<0.0001$ \\
\hline BMI $\left(\mathrm{kg} / \mathrm{m}^{2}\right)$ & $27.7 \pm 4.6$ & $28.7 \pm 4.2$ & $27.72 \pm 4.6$ & $<0.0001$ \\
\hline Smoker & $2291(56.7)$ & $211(61.5)$ & $2080(56.3)$ & 0.06 \\
\hline Diabetes & $490(12.1)$ & $78(22.7)$ & $412(11.1)$ & $<0.0001$ \\
\hline Systolic blood pressure (mmHg) & $131.8 \pm 20.2$ & $142.5 \pm 23.6$ & $131.8 \pm 20.2$ & $<0.0001$ \\
\hline Diastolic blood pressure (mmHg) & $81.3 \pm 10.7$ & $83.8 \pm 12.3$ & $81.3 \pm 10.7$ & 0.0004 \\
\hline Use of antihypertensive medication & $1263(31.3)$ & $163(47.5)$ & $1100(29.8)$ & $<0.0001$ \\
\hline $\log (C A C+1)^{b}$ & $2.3(0 ; 4.5)$ & $4.8(2.7 ; 6.1)$ & $2.3(0 ; 4.5)$ & $<0.0001$ \\
\hline$C A C>0$ & $2734(67.7)$ & $298(86.9)$ & $2436(65.9)$ & $<0.0001$ \\
\hline Use of lipid lowering medication & $360(9.5)$ & $39(12.2)$ & $321(9.3)$ & 0.11 \\
\hline LDL-cholesterol (mg/dL) & $146.8 \pm 36.3$ & $147.9 \pm 34.7$ & $146.8 \pm 36.3$ & 0.40 \\
\hline HDL-cholesterol (mg/dL) & $59.4 \pm 17.1$ & $55.2 \pm 16.8$ & $59.4 \pm 17.1$ & $<0.0001$ \\
\hline Triglycerides (mg/dL) ${ }^{\text {b }}$ & $123.0(89.0 ; 177.0)$ & $141.0(101.0 ; 203.0)$ & $121.0(88.0 ; 175.0)$ & $<0.0001$ \\
\hline Total cholesterol ${ }^{a}$ & $231.4 \pm 38.7$ & $232.2 \pm 39.5$ & $231.3 \pm 38.6$ & 0.73 \\
\hline Coronary artery disease GRS ${ }^{\text {a }}$ & $0.03 \pm 0.003$ & $0.04 \pm 0.003$ & $0.03 \pm 0.003$ & 0.006 \\
\hline
\end{tabular}

$L D L$ low density lipoprotein, $H D L$ high density lipoprotein, $C A C$ coronary artery calcification, GRS genetic risk score. Data are given as number (percentage) unless otherwise indicated

${ }^{\text {a }}$ Data are given as mean $\pm \mathrm{SD}$. ${ }^{\mathrm{b}}$ Data are given as median $(\mathrm{Q} 1 ; \mathrm{Q} 3)$

${ }^{c} p$ are for differences between CHD stratified groups using $\mathrm{X} 2$ or Fisher exact test, $\mathrm{t}$ test or Mann-Whitney $\mathrm{U}$ test 


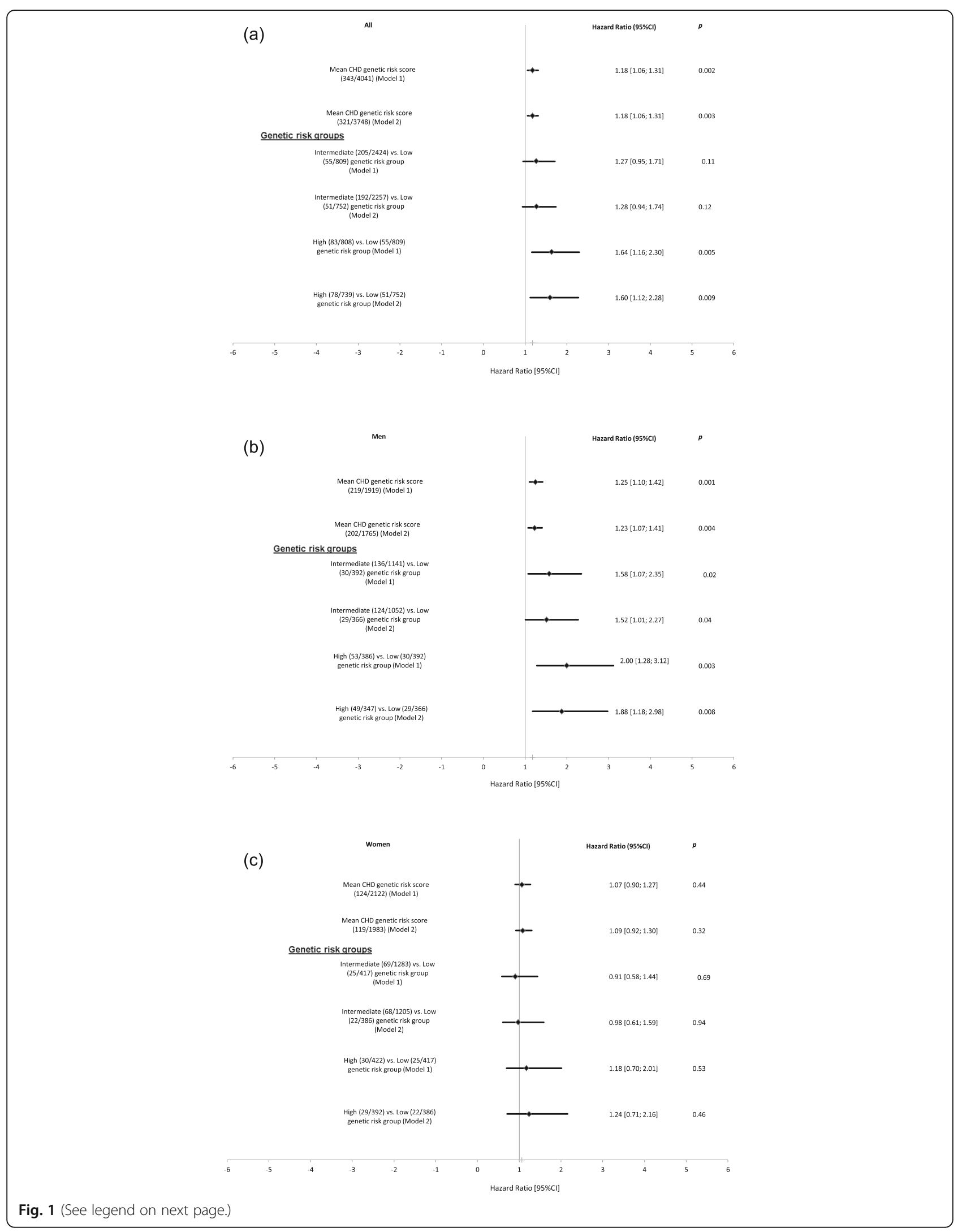


(See figure on previous page.)

Fig. 1 Effect of coronary artery disease genetic risk score with incident coronary heart disease. a all study participants, $\mathbf{b}$ men and $\mathbf{c}$ women. Model 1 is adjusted for age and sex. Model 2 is adjusted for age, sex, smoking, body mass index, diabetes, low density lipoprotein-cholesterol, high density lipoprotein-cholesterol, systolic blood pressure, use of antihypertensive and lipid lowering medication and coronary artery calcification. The numbers in parentheses are given as the (number of events/total number of participants). Additionally, in the sex stratified analyses we excluded the variable sex from the analysis

risk observed in women $\left(\mathrm{HR}_{\text {women }}=1.07\right.$ per-SD [0.90; 1.27], $p=0.44$ ) in the age adjusted analyses (Fig. 1 (b) and (c)).

The HR for incident CHD was statistically significantly higher in the high genetic risk group (high: 1.64 [1.16; 2.30], $p=0.005$ and intermediate: $1.27[0.95 ; 1.71], p=$ 0.11) compared to the low genetic risk group in the age and sex adjusted model. Adjusting for established RFs and CAC did not change the risk estimate (high: 1.60 [1.12; 2.28], $p=0.009$ ) (Fig. 1(a)). Stratifying by sex, the risk was statistically significant for men in the high genetic risk group $(\mathrm{HR}=1.88[1.18 ; 2.98], p=0.008)$ and borderline statistical significant for the intermediate genetic risk group ( $\mathrm{HR}=1.52[1.01 ; 2.27], p=0.04)$ when compared to the low genetic risk group after adjusting for established RFs and CAC (Fig. 1(b)). However, no statistically significant risk was observed for women (high: 1.24 [0.71; 2.16], $p=0.46$ and intermediate: 0.98 [0.61; 1.59], $p=0.94$ ) (Fig. 1(c)). Summary statistics for the association between the individual SNP and incident CHD in the Heinz Nixdorf Recall study participants is shown in the Additional file (Table 1).

We further did CAC stratified analyses $(\mathrm{CAC}=0$ and $\mathrm{CAC}>0$ ) and looked at the association of the genetic risk score with incident $\mathrm{CHD}$ in each stratum adjusting for established CHD RFs (Additional file: Fig. 2 (a-f)). The CAD genetic risk score was statistically significantly associated with incident $\mathrm{CHD}$ only in participants with presence of CAC $(\mathrm{CAC}>0)(\mathrm{HR}=1.21$ per-SD [1.08; 1.36], $p=0.001$ ) (Additional file: Fig. 2(d)). Also, the high genetic risk group with higher $\mathrm{CAC}$ (CAC $>0$ stratum) had statistically significantly higher risk for incident CHD $(\mathrm{HR}=1.70$ [1.16; 2.50], $p=0.007)$. In CAC >0 stratum, statistically significant higher risk was observed only in men in the high genetic risk group $(\mathrm{HR}=2.00$ $[1.23 ; 3.25], p=0.005)$ and showed borderline statistical significance for the intermediate genetic risk group $(\mathrm{HR}=1.52$ [0.98; 2.34], $p=0.06)$ (Additional file: Fig. 2(e)). No statistically significant association was observed for women (Additional file: Fig. 2(f)).

\section{Genetic risk score and coronary artery calcification}

Lastly, we looked at the association between the CAD genetic risk score and CAC. The CAD genetic risk score was statistically significantly associated with CAC. PerSD increase in genetic risk score in the multivariable adjusted model for presence of CAC was OR: 1.19 [1.10;
1.29], $p=1.30 \times 10^{-5}$. Additionally the intermediate (OR: 1.35 [1.10; 1.64], $p=0.004$ ) and higher (OR: 1.62 [1.26; 2.07], $p=0.0002$ ) genetic risk groups showed statistically significant association with presence of CAC when compared with the low genetic risk group. In the sex stratified analyses, CAD genetic risk score showed statistically significantly stronger effects in men in the intermediate (OR: 1.90 [1.39; 2.60], $p=6.4 \times 10^{-5}$ ) and high (OR: 2.37 $[1.56 ; 3.60], p=4.9 \times 10^{-5}$ ) genetic risk groups when compared to the low genetic risk group. No statistically significant risk was observed for women in different genetic risk groups (Table 2).

\section{Discrimination}

Comparing the model consisting of established RFs and $\mathrm{CAC}$ to the model including established RFs and genetic risk score did not reveal improvement of model $(\Delta$ Uno's concordance statistics All $=0.0042 \pm 0.0045, p=$ 0.35) (Additional file: Fig. 3(a)). In the sex stratified analyses, men showed no improvement of the models (RF + CAC and RF + GRS: $\triangle$ Uno's concordance statistics $=-$ $0.0025 \pm 0.0074, p=0.74$ ) (Additional file: Fig. 3(b)). Borderline significant improvement was observed in women when comparing models consisting of established RF and CAC to established RF and genetic risk score $(\Delta$ Uno's concordance statistics $=0.0118 \pm 0.0055, p=0.03$ ) (Additional file: Fig. 3(c)). However, it is to note that both the curves almost overlap each other (Additional file: Fig. 3(c)).

Subsequent AIC values suggested the model consisting of established RFs and CAC in all study participants (AIC value: 4952.851) to be superior compared to the model with established RFs and genetic risk score (AIC value: 4954.940). In sex stratified analyses, the model consisting of established RFs and genetic risk score (AIC value: 2856.279 ) is superior to the model consisting of established RFs and CAC in men (AIC value: 2859.856). However, the model consisting of established RFs and CAC (AIC value: 1673.447) was superior compared to the model with established RFs and genetic risk score in women (AIC value: 1678.255).

\section{Mendelian randomization, with coronary artery disease genetic risk score as the instrumental variable, to assess the causality of CAC for coronary heart disease}

The Mendelian randomization analysis using IVW method showed that genetically predicted CAC is a 
Table 2 Association of coronary artery disease genetic risk score with coronary artery calcification in the Heinz Nixdorf Recall study

\begin{tabular}{|c|c|c|c|c|}
\hline & $\begin{array}{l}\text { Mean GRS } \\
\text { OR }[95 \% \mathrm{Cl}], p\end{array}$ & Low & $\begin{array}{l}\text { Intermediate } \\
\text { OR }[95 \% \mathrm{CI}], p\end{array}$ & $\begin{array}{l}\text { High } \\
\text { OR }[95 \% \mathrm{Cl}], p\end{array}$ \\
\hline \multicolumn{5}{|l|}{ All } \\
\hline$N$ & 4041 & 809 & 2424 & 808 \\
\hline Model 1 & $1.18[1.10 ; 1.27], 6.37 \times 10^{-6}$ & Ref. & $1.29[1.07 ; 1.55], 0.007$ & $1.57[1.25 ; 1.96], 9.7 \times 10^{-5}$ \\
\hline$N$ & 3748 & 752 & 2257 & 739 \\
\hline Model 2 & $1.19[1.10 ; 1.29], 1.30 \times 10^{-5}$ & & $1.35[1.10 ; 1.64], 0.004$ & 1.62 [1.26; 2.07], 0.0002 \\
\hline \multicolumn{5}{|l|}{ Men } \\
\hline$N$ & 1919 & 392 & 1141 & 386 \\
\hline Model 1 & $1.28[1.13 ; 1.44], 5.60 \times 10^{-5}$ & Ref. & $1.67[1.25 ; 2.23], 0.0005$ & $2.17[1.48 ; 3.17], 7.0 \times 10^{-5}$ \\
\hline$N$ & 1765 & 366 & 1052 & 347 \\
\hline Model 2 & $1.29[1.13 ; 1.47], 0.0001$ & & $1.90[1.39 ; 2.60], 6.4 \times 10^{-5}$ & $2.37[1.56 ; 3.60], 4.9 \times 10^{-5}$ \\
\hline \multicolumn{5}{|l|}{ Women } \\
\hline $\mathrm{N}$ & 2122 & 417 & 1283 & 422 \\
\hline Model 1 & $1.13[1.03 ; 1.23], 0.009$ & Ref. & $1.09[0.87 ; 1.38], 0.45$ & $1.30[0.99 ; 1.73], 0.06$ \\
\hline $\mathrm{N}$ & 1983 & 386 & 1205 & 392 \\
\hline Model 2 & 1.13 [1.03; 1.24], 0.01 & Ref. & $1.07[0.83 ; 1.38], 0.59$ & $1.28[0.94 ; 1.76], 0.12$ \\
\hline
\end{tabular}

$\mathrm{N}$ : total number of participants in the analyses. OR odds ratio, [95\% Cl] 95\%confidence interval, Ref reference. Model 1 is adjusted for age and sex. Model 2 is adjusted for age, sex, diabetes, body mass index, systolic blood pressure, smoking, antihypertensive medication, lipid lowering medication, low density lipoprotein-cholesterol and high density lipoprotein-cholesterol

causal risk factor for CHD in men and not in women, with an estimate of 0.90 for the presence of CAC (Estimate $(95 \% \mathrm{CI}), p: 0.90[0.36 ; 1.44], p=0.001$ ) (Table 3).

\section{Discussion}

In the large population-based Heinz Nixdorf Recall study, we investigated the association between CAD genetic risk score and incident CHD. The important findings of our study are i) the CAD genetic risk score was associated with incident $\mathrm{CHD}$, showing stronger effect in men with no effect observed in women, ii) the effect of genetic risk score did not alter even after adjusting for $C A C$, iii) the CAD genetic risk score was associated with incident CHD only in the group with presence of CAC, showing stronger effect in men and no effect in women and iv) CAD genetic risk score was associated with CAC with stronger effect observed in men.

Several large-scale CAD GWAS have led the discovery of novel SNPs [30-33], few of them are recently

Table 3 Causal estimates of the presence of coronary artery calcification on coronary heart disease from Mendelian Randomization analysis

\begin{tabular}{llll}
\hline & CHD & & \\
\cline { 2 - 4 } & Causal estimate & $\mathbf{9 5 \% C l}$ & $\boldsymbol{P}$ \\
\hline IWW (All study participants) & 1.023 & {$[0.38 ; 1.67]$} & 0.002 \\
IWW (Men) & 0.90 & {$[0.36 ; 1.44]$} & 0.001 \\
IWW (Women) & 0.572 & {$[-0.88 ; 2.03]$} & 0.441 \\
\hline
\end{tabular}

IVW inverse-variance weighted, $C H D$ coronary heart disease published and are incorporated in genetic risk score in our study [33]. Similar to previous studies, our study could illustrate that the CAD genetic risk score constructed using 70 SNPs is associated with incident CHD $[11-16,39]$. This effect was more prominent in the higher genetic risk group and especially in men. These results are similar to the findings from a recently published study from MESA, where only men showed higher risk for CHD [11]. CAC, independent of traditional risk factors, is a well know predictor of CHD events [5-7]. $\mathrm{CAC}$ has its own genetic component and is highly heritable $[27,40,41]$. None of the previous studies has used $\mathrm{CAC}$ to find if the association between the CAD genetic risk score and incident $\mathrm{CHD}$ could be altered by CAC. The second finding of our study show that, even after adjusting for RFs and CAC the association between the genetic risk score and incident $\mathrm{CHD}$ remained significant. Moreover, in the CAC stratified analyses the association between the genetic risk score and $\mathrm{CHD}$ was observed only in group with presence of CAC (CAC > $0)$. Especially men belonging to the high genetic risk group were at increased risk of CHD. However, in an observational study from Erbel et al. both men and women belonging to $C A C \geq 400$ group showed similar event rate for men $(8.3 \%)$ and women $(8.2 \%)$ and similar higher risk for CHD [20]. The non-significant association of the genetic risk score in women belonging to the presence of CAC group could be attributed to the different reclassification of $\mathrm{CAC}$ used in the present study i.e. $\mathrm{CAC}=0$ as absence and $\mathrm{CAC}>0$ as presence 
of CAC compared to the previous observational study which used several CAC score risk groups (i.e. low (1$99)$, intermediate (100-399) and high ( $\geq 400)$ risk groups) [20]. Reclassifying men and women based on CAC score into low, intermediate and high risk groups could have yielded different results in the present study. Although the CAD genetic risk score showed strong association with incident CHD in men, comparing the model consisting of established RFs and CAC to the model consisting of RFs and genetic risk score did not improve the risk prediction for CHD when assessed by Uno's concordance. On other hand, risk assessment by Uno's concordance showed positive association in women. These results indicate that model consisting of RFs and CAC is more predictive for women when compared to the model containing RFs and genetic risk score. However, minor but positive results were observed for men in the AIC analyses. Further larger studies are required to evaluate if the risk prediction for CHD could be improved by addition of genetic risk score to the established RFs in men. Furthermore, the results of our study also showed that the CAD genetic risk score was associated with presence of CAC. The results of the combined analyses i.e. using all the study participants are similar to the findings from other studies [42, 43]. However, the sex stratified results showed higher risk only in men. Furthermore, using a Mendelian randomization approach; we found that genetically determined CAC was causally associated with CHD in men.

The present study has several strengths and limitations. The strengths of the study are its longitudinal design, long follow-up time of $11.6 \pm 3.7$ years, the stringent predefined end-point criteria, the external endpoint committee and availability of data on CAC and other CHD established RFs. Along with its strengths the study has its limitations. The greatest limitation of this study is its moderate sample size. Due to its moderate sample size, we could stratify our study population based on CAC score only in two groups $(\mathrm{CAC}=0$ and $\mathrm{CAC}>$ 0 ), however, it would have been more valuable to further stratify the presence of CAC group into low (1-99), intermediate $(100-399)$ and high $(\geq 400)$ risk groups [20]. Larger studies based on presence of CAC stratified analyses could help to find if the genetic risk score is superior to CAC in the risk assessment of CHD in each CAC stratified groups. Furthermore, due to moderate sample size we could not reclassify our study participants based on the recommendations from ACC/AHA Guidelines 2018 [4]. The recommendation suggest the assessment of CAC for treatment decision making in uncertain patients or patients at moderate risk i.e. in patients having atherosclerotic cardiovascular disease risk of $7.5-20 \%$. Further larger studies are required to evaluate the prediction capabilities of the genetic risk score in this uncertain risk group. Also, the results of our study as well as the study from MESA showed similar nonsignificant association of the genetic risk score with $\mathrm{CHD}$ in women, these findings need further investigation. Two important points could be inferred from these findings i) there are other genetic variants for women which are yet to be identified i.e., by conducting sex stratified GWAS as well as by including chromosome $\mathrm{X}$ genetic variants in the GWAS and ii) role of endogenous sex hormones. Studies have shown that premenopausal women when compared to men of the same age have a lower incidence and prevalence of CVD [44, 45]. This sex differences favoring women, seems to disappear after menopause $[46,47]$. Studies have indicated the contribution of reduced levels of ovarian hormones to the higher risk of CVD in women [46, 48-50]. A recent observational study from MESA showed that the postmenopausal women having higher testosterone levels, lower estradiol levels and higher testosterone/estradiol ratio had an elevated risk for CHD events [51]. Further larger studies in women are essential to find if the interaction between the sex hormones and the genetic risk score or genetic variants plays a role in the risk assessment of $\mathrm{CHD}$ in women. Although our results suggest that genetic risk assessment in men in the higher genetic risk groups could be useful, however additional larger studies stratified by sex, different CAC risk groups [20] as well as intermediate risk groups as recommended by ACC/AHA [4] are needed to evaluate the usefulness of genetic risk score for assessment of CHD in men.

\section{Conclusions}

The findings of our study suggest that the CAD genetic risk score could be useful for CHD risk prediction, at least in men belonging to the higher genetic risk group, but it does not outbalance the value of the CT-based quantification of CAC which works independently on both men as well as women and allows better risk stratification in both the genders.

\section{Supplementary information}

Supplementary information accompanies this paper at https://doi.org/10. 1186/s12881-020-01113-y.

\section{Additional file 1.}

\footnotetext{
Abbreviations

CAD: Coronary artery disease; CHD: Coronary heart disease; CAC: coronary artery calcification; Q/q: Quintiles; RF: Risk factor; CT: Computer tomography; ACC/AHA: American College of Cardiology/American Heart Association; MESA: Multi Ethnic Study of Atherosclerosis; GWAS: genome-wide association studies; SNP: single nucleotide polymorphism; BMI: body mass index; LDL: Iow density lipoprotein; HDL: high density lipoprotein; AIC: Akaike's information criterion; IVW: inverse-variance weighted
} 


\section{Acknowledgements}

We are indebted to all the study participants and to the dedicated personnel of both the study center of the Heinz Nixdorf Recall study and the EBTscanner facilities D. Grönemeyer, Bochum, and R. Seibel, Mülheim, as well as to the investigative group, in particular to U. Roggenbuck, U. Slomiany, E. M. Beck, M. Bauer, A. Öffner, S. Münkel, S. Schrader, R. Peter, and H. Hirche. Scientific advisory board: T. Meinertz, Hamburg (Chair); M. Blettner, Mainz; C. Bode, Freiburg; P. J. de Feyter, Rotterdam, Zürich, Niederlande; B. Güntert, Hall i.T., Schweiz; F. Gutzwiller, Schweiz; H. Heinen, Bonn; O. Hess”, Bern, Schweiz; B. Klein ${ }^{\ddagger}$, Essen; H. Löwel, Neuherberg; M. Reiser, München; G. Schmidt ${ }^{\ddagger}$, Essen; M. Schwaiger, München; C. Steinmüller, Bonn; T. Theorell, Stockholm, Schweden; S. N. Willich, Berlin. Criteria and end-point committee: C. Bode, Freiburg (Chair), K. Berger, Münster; H. R. Figulla, Jena; C. Hamm, Bad Nauheim; P. Hanrath, Aachen; W. Köpcke, Münster; C. Weimar, Essen; A. Zeiher, Frankfurt. Technical support for the imputation of the Heinz Nixdorf Recall Study data on the Supercomputer Cray XT6m was provided by the Center for Information and Media Services, University of Duisburg-Essen.

\section{Authors' contributions}

Writing team: SP; all the authors reviewed, edited and approved the manuscript; conceptualization: SP, K-HJ, RE, SM; investigation: KHJ, RE, SM; acquisition of data: $K H J, R E$, SM; statistical analyses: SP; methodology: KHJ, RE, SM, NL; genotyping and imputations: PH, MMN, SP; project administration: SP; supervision: NL, KHJ, RE, SM.

\section{Funding}

We thank the Heinz Nixdorf Foundation [Chairman: M. Nixdorf; Past Chairman: G. Schmidt ${ }^{\ddagger}$, the "Deutsche Forschungsgemeinschaft" (Project numbers: PE 2309/2-1, ER 155/6-1 and ER 155/6-2) and the "Bundesministerium für Bildung und Forschung" for the generous support of this study. We thank the Ministry of Innovation, Science and Research, Nordrhine-Westfalia for the generous support for the genotyping of the Heinz Nixdorf Recall study participants. An additional research grant was received from Imatron Inc., South San Francisco, CA, which produced the EBCT scanners, and GE-Imatron, South San Francisco, CA, after the acquisition of Imatron Inc. We acknowledge the support of the Sarstedt AG \& Co. (Nümbrecht, Germany) concerning laboratory equipment. There is no role of any funding body in the design of the study and collection, analysis, and interpretation of data and writing of manuscript. Open Access funding provided by Projekt DEAL.

\section{Availability of data and materials}

Due to data security reasons i.e. the data contain potentially participant identifying information, the Heinz Nixdorf Recall study does not allow sharing data as a public use file. However, other authors/researchers are allowed to access data upon request, which is the same way the authors of the present paper obtained the data. Data requests can be addressed to recall@uk-essen.de.

\section{Ethics approval and consent to participate}

The study has been approved by the ethical committee at the University Hospital Essen, Germany and is conducted in accordance with the principles expressed in the Declaration of Helsinki. The study was certified and recertified according to DIN EN ISO 9001:2000/2008. All study participants gave their written informed consent. In order to access the data for the present study, the authors have obtained permission from the internal committee of the Heinz Nixdorf Recall study.

\section{Consent for publication}

Not applicable.

\section{Competing interests}

The authors declare that they have no competing interests.

\section{Author details}

${ }^{1}$ Institute for Medical Informatics, Biometry and Epidemiology, University Hospital of Essen, University Duisburg-Essen, Essen, Germany. ${ }^{2}$ Department of Genomics, Life \& Brain Center, University of Bonn, Bonn, Germany. ${ }^{3}$ Division of Medical Genetics, Department of Biomedicine, University of Basel, Basel, Switzerland. ${ }^{4}$ Centre for Urban Epidemiology, University Hospital Essen, Essen, Germany.
Received: 14 February 2020 Accepted: 31 August 2020

Published online: 10 September 2020

\section{References}

1. Wilkins E, Wilson L, Wickramasinghe K, Bhatnagar P, Leal J, LuengoFernandez R, Burns R, Rayner M, Townsend N. European Cardiovascular Disease Statistics 2017. European Heart Network Brussels. 2017. http://www. ehnheart.org/cvd-statistics/cvd-statistics-2017.html.

2. Benjamin EJ, Virani SS, Callaway CW, Chamberlain AM, Chang AR, Cheng S, et al. Heart disease and stroke Statistics-2018 update: a report from the American Heart Association. Circulation. 2018;137:e67-e492. https://doi.org/ 10.1161/CIR.0000000000000558.

3. Piepoli MF, Hoes AW, Agewall S, Albus C, Brotons C, Catapano AL, et al. 2016 European Guidelines on cardiovascular disease prevention in clinical practice: The Sixth Joint Task Force of the European Society of Cardiology and Other Societies on Cardiovascular Disease Prevention in Clinical Practice (constituted by representatives of 10 societies and by invited experts) Developed with the special contribution of the European Association for Cardiovascular Prevention \& Rehabilitation (EACPR). Atherosclerosis. 2016; 252:207-74. https://doi.org/10.1016/j.atherosclerosis.2016.05.037.

4. Grundy SM, Stone NJ, Bailey AL, Beam C, Birtcher KK, Blumenthal RS, et al. 2018 AHA/ACC/AACVPR/AAPA/ABC/ACPM/ADA/AGS/APhA/ASPC/NLA/ PCNA guideline on the Management of Blood Cholesterol: executive summary: a report of the American College of Cardiology/American Heart Association task force on clinical practice guidelines. J Am Coll Cardiol. 2018. https://doi.org/10.1016/j.jacc.2018.11.002.

5. Erbel R, Budoff M. Improvement of cardiovascular risk prediction using coronary imaging: subclinical atherosclerosis: the memory of lifetime risk factor exposure. Eur Heart J. 2012;33:1201-13. https:/doi.org/10.1093/eurhearti/ehs076.

6. McClelland RL, Jorgensen NW, Budoff M, Blaha MJ, Post WS, Kronmal RA, et al. 10-year coronary heart disease risk prediction using coronary artery calcium and traditional risk factors: derivation in the MESA (multi-ethnic study of atherosclerosis) with validation in the HNR (Heinz Nixdorf recall) study and the DHS (Dallas heart study). J Am Coll Cardiol. 2015;66:1643-53. https://doi.org/10.1016/j.jacc.2015.08.035.

7. Möhlenkamp S, Lehmann N, Moebus S, Schmermund A, Dragano N, Stang A, et al. Quantification of coronary atherosclerosis and inflammation to predict coronary events and all-cause mortality. J Am Coll Cardiol. 2011;57: 1455-64. https://doi.org/10.1016/j.jacc.2010.10.043.

8. Marenberg ME, Risch N, Berkman LF, Floderus B, de Faire U. Genetic susceptibility to death from coronary heart disease in a study of twins. N Engl J Med. 1994;330:1041-6. https://doi.org/10.1056/ NEJM199404143301503.

9. Zdravkovic S, Wienke A, Pedersen NL, Marenberg ME, Yashin Al, de Faire U. Heritability of death from coronary heart disease: a 36-year follow-up of 20 966 Swedish twins. J Intern Med. 2002;252:247-54.

10. Won H-H, Natarajan P, Dobbyn A, Jordan DM, Roussos P, Lage K, et al. Disproportionate contributions of select genomic compartments and cell types to genetic risk for coronary artery disease. PLoS Genet. 2015;11: e1005622. https://doi.org/10.1371/journal.pgen.1005622.

11. Hajek C, Guo X, Yao J, Hai Y, Johnson WC, Frazier-Wood AC, et al. Coronary heart disease genetic risk score predicts cardiovascular disease risk in men. Not Women Circ Genom Precis Med. 2018;11:e002324. https://doi.org/10. 1161/CIRCGEN.118.002324.

12. Iribarren C, Lu M, Jorgenson E, Martínez M, Lluis-Ganella C, Subirana I, et al. Clinical utility of multimarker genetic risk scores for prediction of incident coronary heart disease: a cohort study among over 51 thousand individuals of European ancestry. Circ Cardiovasc Genet. 2016;9:531-40. https://doi.org/ 10.1161/CIRCGENETICS.116.001522.

13. Iribarren C, Lu M, Jorgenson E, Martínez M, Lluis-Ganella C, Subirana I, et al. Weighted multi-marker genetic risk scores for incident coronary heart disease among individuals of African. Latino East-Asian Ancestry Sci Rep. 2018;8:6853. https://doi.org/10.1038/s41598-018-25128-x.

14. Krarup NT, Borglykke A, Allin KH, Sandholt CH, Justesen JM, Andersson EA, et al. A genetic risk score of 45 coronary artery disease risk variants associates with increased risk of myocardial infarction in 6041 Danish individuals. Atherosclerosis. 2015;240:305-10. https://doi.org/10.1016/j. atherosclerosis.2015.03.022.

15. Ripatti S, Tikkanen E, Orho-Melander M, Havulinna AS, Silander K, Sharma A, et al. A multilocus genetic risk score for coronary heart disease: case-control 
and prospective cohort analyses. Lancet. 2010;376:1393-400. https://doi.org/ 10.1016/S0140-6736(10)61267-6.

16. Tada H, Melander O, Louie JZ, Catanese JJ, Rowland CM, Devlin JJ, et al. Risk prediction by genetic risk scores for coronary heart disease is independent of self-reported family history. Eur Heart J. 2016;37:561-7. https://doi.org/10.1093/ eurheartj/ehv462.

17. Schmermund A, Möhlenkamp S, Stang A, Grönemeyer D, Seibel R, Hirche H, et al. Assessment of clinically silent atherosclerotic disease and established and novel risk factors for predicting myocardial infarction and cardiac death in healthy middleaged subjects: rationale and design of the Heinz Nixdorf RECALL study. Risk factors, evaluation of coronary calcium and lifestyle. Am Heart J. 2002;144:212-8.

18. Schmermund A, Möhlenkamp S, Berenbein S, Pump H, Moebus S, Roggenbuck U, et al. Population-based assessment of subclinical coronary atherosclerosis using electron-beam computed tomography. Atherosclerosis. 2006;185:177-82. https://doi.org/10.1016/.atherosclerosis.2005.06.003.

19. Lehmann N, Erbel R, Mahabadi AA, Rauwolf M, Möhlenkamp S, Moebus S, et al. Value of progression of coronary artery calcification for risk prediction of coronary and cardiovascular events: result of the HNR study (Heinz Nixdorf recall). Circulation. 2018;137:665-79. https://doi.org/10.1161/ CIRCULATIONAHA.116.027034.

20. Erbel R, Möhlenkamp S, Moebus S, Schmermund A, Lehmann N, Stang A, et al. Coronary risk stratification, discrimination, and reclassification improvement based on quantification of subclinical coronary atherosclerosis: the Heinz Nixdorf recall study. J Am Coll Cardiol. 2010;56: 1397-406. https://doi.org/10.1016/j.jacc.2010.06.030.

21. Lehmann N, Erbel R, Mahabadi AA, Kälsch H, Möhlenkamp S, Moebus S, et al. Accelerated progression of coronary artery calcification in hypertension but also prehypertension. J Hypertens. 2016;34:2233-42. https://doi.org/10.1097/HJH.0000000000001080.

22. Agatston AS, Janowitz WR, Hildner FJ, Zusmer NR, Viamonte M, Detrano R. Quantification of coronary artery calcium using ultrafast computed tomography. J Am Coll Cardiol. 1990;15:827-32. https://doi.org/10.1016/0735-1097(90)90282-t.

23. Lehmann N, Möhlenkamp S, Mahabadi AA, Schmermund A, Roggenbuck U, Seibel $R$, et al. Effect of smoking and other traditional risk factors on the onset of coronary artery calcification: results of the Heinz Nixdorf recall study. Atherosclerosis. 2014;232: 339-45. https:/doi.org/10.1016/.atherosclerosis.2013.11.045.

24. Stang A, Moebus S, Möhlenkamp S, Dragano N, Schmermund A, Beck E-M, et al. Algorithms for converting random-zero to automated oscillometric blood pressure values, and vice versa. Am J Epidemiol. 2006;164:85-94. https://doi.org/10.1093/aje/kwj160.

25. Moebus S, Stang A, Möhlenkamp S, Dragano N, Schmermund A, Slomiany $\mathrm{U}$, et al. Association of impaired fasting glucose and coronary artery calcification as a marker of subclinical atherosclerosis in a population-based cohort--results of the Heinz Nixdorf recall study. Diabetologia. 2009;52:81-9. https://doi.org/10.1007/s00125-008-1173-y.

26. Geisel MH, Coassin S, Heßler N, Bauer M, Eisele L, Erbel R, et al. Update of the effect estimates for common variants associated with carotid intima media thickness within four independent samples: the Bonn IMT family study, the Heinz Nixdorf recall study, the SAPHIR study and the Bruneck study. Atherosclerosis. 2016;249:83-7. https://doi.org/10.1016/.jatherosclerosis.2016.03.042.

27. Pechlivanis S, Mühleisen TW, Möhlenkamp S, Schadendorf D, Erbel R, Jöckel $\mathrm{K}-\mathrm{H}$, et al. Risk loci for coronary artery calcification replicated at 9p21 and 6q24 in the Heinz Nixdorf recall study. BMC Med Genet. 2013;14:23. https:// doi.org/10.1186/1471-2350-14-23.

28. Pechlivanis S, Heilmann-Heimbach S, Erbel R, Mahabadi AA, Hochfeld LM, Jöckel K-H, et al. Male-pattern baldness and incident coronary heart disease and risk factors in the Heinz Nixdorf recall study. PLoS One. 2019;14: e0225521. https://doi.org/10.1371/journal.pone.0225521.

29. Pechlivanis S, Mahabadi AA, Hoffmann P, Nöthen MM, Broecker-Preuss M, Erbel $R$, et al. Association between lipoprotein(a) (Lp(a)) levels and $L p(a)$ genetic variants with coronary artery calcification. BMC Med Genet. 2020;21: 62. https://doi.org/10.1186/s12881-020-01003-3.

30. Schunkert H, König IR, Kathiresan S, Reilly MP, Assimes TL, Holm H, et al. Largescale association analysis identifies 13 new susceptibility loci for coronary artery disease. Nat Genet. 2011;43:333-8. https://doi.org/10.1038/ng.784.

31. Nikpay M, Goel A, Won H-H, Hall LM, Willenborg C, Kanoni S, et al. A comprehensive 1,000 genomes-based genome-wide association meta-analysis of coronary artery disease. Nat Genet. 2015;47:1121-30. https://doi.org/10.1038/ng.3396.

32. Deloukas P, Kanoni S, Willenborg C, Farrall M, Assimes TL, Thompson JR, et al. Large-scale association analysis identifies new risk loci for coronary artery disease. Nat Genet. 2013;45:25-33. https://doi.org/10.1038/ng.2480.
33. Webb TR, Erdmann J, Stirrups KE, Stitziel NO, Masca NGD, Jansen H, et al. Systematic evaluation of Pleiotropy identifies 6 further loci associated with coronary artery disease. J Am Coll Cardiol. 2017;69:823-36. https://doi.org/ 10.1016/j.jacc.2016.11.056.

34. Pechlivanis S, Moebus S, Lehmann N, Erbel R, Mahabadi AA, Hoffmann P, et al. Genetic risk scores for coronary artery disease and its traditional risk factors: their role in the progression of coronary artery calcification-results of the Heinz Nixdorf recall study. PLoS One. 2020. https://doi.org/10.1371/ journal.pone.0232735.

35. Purcell $S$, Neale B, Todd-Brown K, Thomas L, Ferreira MAR, Bender D, et al. PLINK: a tool set for whole-genome association and population-based linkage analyses. Am J Hum Genet. 2007;81:559-75. https://doi.org/10.1086/ 519795.

36. Uno H, Cai T, Pencina MJ, D'Agostino RB, Wei LJ. On the C-statistics for evaluating overall adequacy of risk prediction procedures with censored survival data. Stat Med. 2011;30:1105-17. https://doi.org/10.1002/sim.4154.

37. Akaike H. Information Theory and an Extension of the Maximum Likelihood Principle. Second International Symposium on Information Theory Budapest Akademiai Kiado;1973:267-281.

38. Yavorska OO, Burgess S. MendelianRandomization: an R package for performing Mendelian randomization analyses using summarized data. Int J Epidemiol. 2017;46:1734-9. https://doi.org/10.1093/ije/dyx034.

39. Morrison AC, Bare LA, Chambless LE, Ellis SG, Malloy M, Kane JP, et al. Prediction of coronary heart disease risk using a genetic risk score: the atherosclerosis risk in communities study. Am J Epidemiol. 2007;166:28-35. https://doi.org/10.1093/aje/kwm060.

40. O'Donnell CJ, Kavousi M, Smith AV, Kardia SLR, Feitosa MF, Hwang S-J, et al. Genome-wide association study for coronary artery calcification with followup in myocardial infarction. Circulation. 2011;124:2855-64. https://doi.org/10 1161/CIRCULATIONAHA.110.974899.

41. Peyser PA, Bielak LF, Chu JS, Turner ST, Ellsworth DL, Boerwinkle E, Sheedy PF. Heritability of coronary artery calcium quantity measured by electron beam computed tomography in asymptomatic adults. Circulation. 2002;106:304-8.

42. Natarajan P, Young R, Stitziel NO, Padmanabhan S, Baber U, Mehran R, et al. Polygenic risk score identifies subgroup with higher burden of atherosclerosis and greater relative benefit from statin therapy in the primary prevention setting. Circulation. 2017;135:2091-101. https://doi.org/ 10.1161/CIRCULATIONAHA.116.024436.

43. van Setten J, Išgum I, Pechlivanis S, Tragante V, de Jong PA, Smolonska J, et al. Serum lipid levels, body mass index, and their role in coronary artery calcification: a polygenic analysis. Circ Cardiovasc Genet. 2015;8:327-33. https://doi.org/10.1161/CIRCGENETICS.114.000496.

44. Kavousi M. Differences in epidemiology and risk factors for atrial fibrillation between women and men. Front Cardiovasc Med. 2020;7:3. https://doi.org/ 10.3389/fcrm.2020.00003.

45. Wake R, Yoshiyama M. Gender differences in ischemic heart disease. Recent Pat Cardiovasc Drug Discov. 2009;4:234-40. https://doi.org/10.2174/ 157489009789152249.

46. Bittner V. Menopause, age, and cardiovascular risk: a complex relationship. J Am Coll Cardiol. 2009;54:2374-5. https://doi.org/10.1016/j.jacc.2009.10.008.

47. Yang X-P, Reckelhoff JF. Estrogen, hormonal replacement therapy and cardiovascular disease. Curr Opin Nephrol Hypertens. 2011;20:133-8. https:// doi.org/10.1097/MNH.0b013e3283431921.

48. Spoletini I, Vitale C, Pelliccia F, Fossati C, Rosano GMC. Androgens and cardiovascular disease in postmenopausal women: a systematic review. Climacteric. 2014;17:625-34. https://doi.org/10.3109/13697137.2014. 887669.

49. Morselli E, Santos RS, Criollo A, Nelson MD, Palmer BF, Clegg DJ. The effects of oestrogens and their receptors on cardiometabolic health. Nat Rev Endocrinol. 2017;13:352-64. https://doi.org/10.1038/nrendo.2017.12.

50. Regitz-Zagrosek V, Kararigas G. Mechanistic pathways of sex differences in cardiovascular disease. Physiol Rev. 2017;97:1-37. https://doi.org/10.1152/ physrev.00021.2015.

51. Zhao D, Guallar E, Ouyang P, Subramanya V, Vaidya D, Ndumele CE, et al. Endogenous sex hormones and incident cardiovascular disease in Postmenopausal women. J Am Coll Cardiol. 2018;71:2555-66. https://doi.org/10. 1016/j.jacc.2018.01.083.

\section{Publisher's Note}

Springer Nature remains neutral with regard to jurisdictional claims in published maps and institutional affiliations. 